\title{
Roles of Circular RNAs And Their Interactions With MicroRNAs in Human Disorders
}

\author{
Jun-feng Sun ${ }^{\mathrm{a}}$, Su-ling Wu ${ }^{\mathrm{b}}$, Guo-jun Wang ${ }^{\mathrm{a}}$, Jia-xiang Wang ${ }^{\mathrm{a} *}$ \\ ${ }^{a}$ Department of Surgery, The First Affiliated Hospital of Zhengzhou University, Zhengzhou, 450052, China. \\ ${ }^{b}$ Department of Pediatrics, The people's hospital of Yucheng county in Henan province, Shangqiu, 476300, China.
}

\begin{abstract}
Circular RNAs (circRNAs) are a class of non-coding RNAs (ncRNAs) that are involved in transcriptional and posttranscriptional gene expression regulation by sponging microRNAs (miRNAs). As miRNAs control a large set of biological processes, circRNA sponge activity also affects these pathways and thereby participates in human disease initiation and development. A growing number of works have confirmed that circRNAs may play critical roles in normal human tissues and organ functions and in the pathogenesis of human diseases, mainly by interacting with miRNAs. Herein, we review the expressions and functions of circRNAs in diverse disorders, including those of the nervous system and cardiovascular system, cancer, and other common diseases. As circRNAs are easily detected in many clinical samples, they present great potential as biomarkers for disease diagnosis and prognostic evaluation; thus, the prospect of using circRNAs as biomarkers for clinical decision-making is also discussed.
\end{abstract}

Keywords: circular RNAs; miRNAs; neurological disorders; cancer; cardiovascular diseases; biomarker

\section{INTRODUCTION}

Circular RNAs (circRNAs) have recently been identified as a novel class of non-coding RNAs (ncRNAs) that are more stable than linear RNAs because of their unique closed loop structure, with no $5^{\prime}$ to $3^{\prime}$ polarity or polyadenylated tail ${ }^{[1]}$. They are generated from either exons or introns via alternative back-splicing, with an upstream splice acceptor joined to a downstream splice donor ${ }^{[2]}$. Reverse complementary sequences or RNA-binding proteins are necessary for circRNA biogenesis. Emerging evidence reveals that the majority of circRNAs are evolutionarily conserved across species and specifically expressed in distinctive tissues and developmental stages ${ }^{[3]}$. The dysregulation and abnormal expression of circRNAs have been implicated in disease development and progression ${ }^{[4]}$, implying their potential role in pathogenesis.

MicroRNAs (miRNAs) are another type of ncRNAs that negatively regulate gene expression at the post-transcription level by binding to the $3^{\prime}$ UTR of their target mRNAs to participate in multiple biological processes. However, competitive endogenous RNAs (ceRNAs) of

\footnotetext{
*Corresponding author: Jia-xiang Wang

Mailing address: Department of Surgery, The First Affiliated Hospital of Zhengzhou University, Jianshe East Road NO.1, Zhengzhou, Henan 450052, People's Republic.

E-mail: wangzhiwei126@126.com

Received: 20 April 2018 Accepted: 26 June 2018
}

miRNAs, such as the long non-coding RNAs (lncRNAs), devote themselves to binding with miRNAs and thus relieving their inhibitory effects on their target genes ${ }^{[5]}$. Similarly to other ceRNAs, circRNAs also serve as miRNA sponges and cause miRNA deregulation that affects cell function ${ }^{[6]}$. It has been widely validated that endogenous circRNAs work as miRNA sponges to rescue their target genes, forming a circRNA-miRNA-mRNA axis to modulate numerous signaling pathways. Considering the enormous number of miRNAs and their target mRNAs encoded in human genome, their interactions and the regulation mediated by circRNAs have attracted extensive attention in human disorders. Therefore, the roles that circRNAs play in human disorders by interacting with miRNAs are reviewed in this article. CircRNAs' potential as biomarkers for clinical diagnosis and prognosis is also emphasized.

\section{CIRCRNAS IN NEUROLOGICAL DIS- ORDERS}

CircRNAs are pivotal in neurodevelopment and are reported to be highly enriched in the nervous system and notably upregulated during neuronal differentiation ${ }^{[7,8]}$, hinting at their potential neural function. The much-studied circRNA cerebellar degeneration-related protein 1 antisense (CDR1as) is a well-known miR-7 sponge that has over 60 binding sites for miR-7 (also called ciRS-7) ${ }^{[9]}$ (Figure 1) and has been linked to nervous system diseases. Through 
glioblastoma multiforme (GBM) biopsies, CDR1as was found to manifest as a downstream miR-671-5p target in human GBM, an aggressive cancer originating from the central nervous system. The expression of CDR1as was also found to be negatively correlated with that of miR-671-5p ${ }^{[10]}$. In the sporadic Alzheimer's disease (AD) hippocampal CAI region, a dysregulated miR7-ciRS-7 system has been noted ${ }^{[11]}$. Deficits in ciRS7 and ciRS-7 sponging activities were expected to elevate ambient miR-7 levels in brain cells affected by $\mathrm{AD}$, contributing to the downregulation of the mRNA targets of miR-7, such as the ubiquitin protein ligase $A$ (UBE2A).

CDR1as has also been proven to be expressed mostly in the brain and has been associated with the neurological disorder Parkinson's disease, which results from the degeneration of dopaminegenerating cells in the midbrain ${ }^{[12]}$. Recently, a major breakthrough was made in demonstrating an in vivo loss-of-function circRNA phenotype in mice ${ }^{[13]}$. When the CDR1as locus was knocked out from the mouse genome, the animals displayed sensorimotor gating deficits and a behavioral phenotype involving failure to filter out unnecessary information, manifesting as neuropsychiatric disorders. This was the first time the function of a circRNA was verified in vivo, highlighting the roles of circRNAs in normal brain function.

In addition to CDR1as, circRNAs are abundant in the brains of mammals, and their ability to transverse the blood-brain barrier makes them good candidate regulators in central nervous system disorders. In mouse and human brain samples, the 15849 and 65731 circRNA candidates have been respectively determined, with distinct spatio-temporal expression patterns ${ }^{[8]}$. In spared nerve injury-induced neuropathic pain, 188 circRNAs were found to be markedly upor downregulated 14 days after spared nerve injury surgery, facilitating the development of promising neuropathic pain therapeutics targeting circRNAs ${ }^{[14]} \mathrm{JIn}$ cerebral ischemia-reperfusion injuries (IRI) induced by oxygen-glucose deprivation/reoxygenation (OGD/R), the significant upregulation of mmu-circRNA-015947 and its interaction with five miRNA targets were verified, showing that this circRNA plays a crucial role in OGD/ R-induced neuron injury by binding with miRNAs ${ }^{[15]}$. A recently identified circRNA, hsa_circ_0021001, was confirmed to be significantly decreased in the peripheral blood of intracranial aneurysm patients as compared to that of controls, demonstrating its great effectiveness in the diagnosis of intracranial aneurysms [16]. The circRNAs involved in neurological disorders and their up- or downstream targets are presented in Table 1. The functions in vivo of these circRNAs require further confirmation, and more decisive identifications of the circRNAs involved in neurological disorders are expected in the future.

\section{CIRCRNAS IN CANCERS}

The detection of increased circRNAs in the peripheral blood, sera, and tissues of cancer patients have been widely reported (Table 2). A circular, testisspecific sense transcript of the sex-determining region Y (SRY) gene, also named SRY, acts to control the biological effects of miR-138 by binding to its 16 conserved binding sites [17]. In cholangiocarcinoma, SRY suppresses the expression of miR-138, leading to decreased mRNA and protein levels for the Ras homolog gene family member $\mathrm{C}$ (RhoC). This finally promotes the proliferation, migration, and invasion of cholangiocarcinoma cells ${ }^{[18]}$. CDR1as was shown to be upregulated in hepatocellular carcinoma (HCC) tissues, and the knockdown of CDR1as was shown to repress HCC cell proliferation and invasion by targeting miR$7^{[19]}$, suggesting that CDR1as has an oncogenic effect in HCC ${ }^{[20,21]}$. Through targeting miR-7, CDR1as also influences the progression of colorectal cancer, and it was found to be positively related to tumor size, $\mathrm{T}$ stage, lymph node metastasis, and poor overall patient

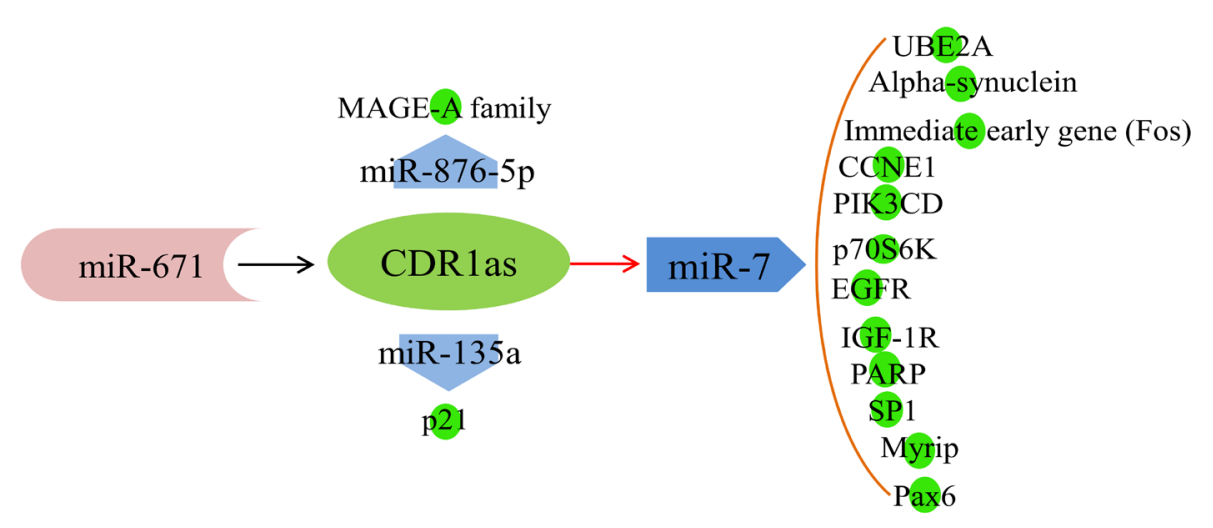

Figure 1. CircRNA CDR1as and its interaction with miRNAs. 
Table 1. CircRNAs identified in neurological disorders.

\begin{tabular}{|c|c|c|c|c|c|}
\hline Disorders & Sites or samples & CircRnAs & MiRNAs & Potential targets & References \\
\hline GBM & Biopsies & CDR1as & miR-671-5p1 & - & {$[10]$} \\
\hline $\mathrm{AD}$ & Hippocampal CAI region & CDR1as & miR-7 & UBE2A & {$[11]$} \\
\hline Parkinson's disease & Midbrain & CDR1as & miR-7 & Alpha-synuclein & {$[12]$} \\
\hline Neuropsychiatric disorder & In vivo & CDR1as & miR-7 & Immediate early gene (Fos) & {$[13]$} \\
\hline OGD/R-induced neuron injury & HT22 cells & mmu-circRNA-015947 & $\begin{array}{l}\text { 2miR-188-3p, } \\
\text { miR-329-5p, } \\
\text { miR-3057-3p, } \\
\text { miR-5098, } \\
\text { miR-683 }\end{array}$ & PSMG3 & {$[15]$} \\
\hline Intracranial aneurysms & Peripheral blood & hsa_circ_0021001 & - & - & {$[16]$} \\
\hline
\end{tabular}

1CDR1as is the downstream target of miR-671-5p. 2These refer to miRNAs in mice.

GBM, glioblastoma multiforme; AD, Alzheimer's disease; OGD/R, oxygen-glucose deprivation/reoxygenation; CDR1as, cerebellar degeneration-related protein 1 antisense; UBE2A, ubiquitin protein ligase A; PSMG3, Proteasome assembly chaperon 3

survival ${ }^{[22]}$.

By acting as a miR-876-5p sponge to boost the melanoma-antigen family A (MAGE-A) expression, CDR1as also accelerates esophageal squamous cell carcinoma progression ${ }^{[23]}$. This effect is distinct from the inhibitory effect of circ-IICH on this cancer ${ }^{[24]}$. In addition, one recent study proclaimed that CDR1as exhibited anti-oncogenic functions in bladder cancer by sponging miR-135a. In this study, a downregulation of CDR1as was observed, and the overexpression of CDR1as suppressed the proliferation, invasion, and migration of bladder cancer cells in vitro and retarded tumor growth in vivo ${ }^{[25]}$. In cholangiocarcinoma, the expression of CDR1as was notably higher than that in adjacent normal tissues, and it was closely associated with lymph node invasion, advanced tumor node metastasis (TNM) stage, and postoperative recurrence, implying its involvement in the oncogenesis and metastasis of cholangiocarcinoma ${ }^{[26]}$.

In osteosarcoma cell lines, tissues, and plasma, circHIPK3 was found to be stably downregulated, and this downregulation was associated with poor prognosis. In addition, circ-HIPK3 overexpression notably inhibited the proliferation, invasion, and migration of osteosarcoma cells ${ }^{[27]}$. Screening differential circRNA expression profiles also revealed the regulatory role of circTCF25 in bladder carcinoma ${ }^{[28]}$. Analyses of gastric cancer tissues from gastric cancer surgery patients and plasma samples from preoperative and postoperative gastric cancer patients revealed remarkably low expressions of hsa_circ_002059. Low hsa_circ_002059 expression was significantly associated with advanced TNM stage, distal metastasis, male, and age ${ }^{[29]}$. CircRNA_100269 was also verified as being downregulated in gastric cancer and as suppressing tumor cell growth by targeting miR-630 ${ }^{[30]}$. In human oral squamous cell carcinomas, circRNA_100290 was shown to function as a competing endogenous RNA to modulate CDK6 expression via the sponging of miR-29b family members ${ }^{[11]}$. Similarly to CDR1as, circ_001569 was also shown to be upregulated in colorectal cancer and correlated with its aggressive characteristics ${ }^{[32]}$, while its interaction with miRNAs warrants future clarification.

\section{CIRCRNAS IN CARDIOVASCULAR DIS- EASES}

Cardiovascular diseases related to stroke and coronary heart disease are increasingly recognized as leading causes of premature mortality. Hence, therapeutic intervention and early prevention are urgently needed to address this issue ${ }^{[33]}$. In the diabetic mouse myocardium, 45 circRNAs were found to be increased, while 31 were decreased. Among these, circRNA_000203 was shown to be remarkably upregulated and to contribute to myocardial fibrosis by restraining the function of miR-26b-5p [34]. By controlling the miR-141/TGF- $\beta 1$ signaling axis, circRNA_010567 presented a similar impact on myocardial fibrosis progression ${ }^{[35]}$. In the peripheral blood of patients with acute myocardial infarction, 1670 circRNAs and 13 miRNAs showed differential expressions, and multiple circRNA-miRNA interactions were implicated in the occurrence of acute myocardial infarction ${ }^{[36]}$. An in silico approach identified decreased expression of a myocardial infarction-associated circRNA (MICRA) in the peripheral blood of patients with myocardial infarction. Low MICRA expression predicted left ventricular dysfunction ${ }^{[37]}$, and MICRA also improved risk classification after myocardial infarction ${ }^{[38]}$. The CDR1as/miR-7 pathway also plays a role in inducing cardiomyocyte apoptosis to promote 
Table 2. Roles of circRNAs in diverse cancers.

\begin{tabular}{|c|c|c|c|c|c|}
\hline & Sites or samples & CircRnAs & Target miRNAs & Potential targets & References \\
\hline Cholangiocarcinoma & Cells & SRY & miR-138 & RhoC & [18] \\
\hline \multirow[t]{3}{*}{ Hepatocellular carcinoma } & \multirow[t]{3}{*}{ Tissues and cells } & \multirow[t]{3}{*}{ CDR1as } & \multirow[t]{3}{*}{ miR-7 } & CCNE1, PIK3CD & [19] \\
\hline & & & & p70s6K & {$[20]$} \\
\hline & & & & EGFR & {$[21]$} \\
\hline \multirow{2}{*}{ Colorectal cancer } & Tissues and cells & CDR1as & $\operatorname{miR}-7$ & EGFR, IGF-1R & {$[22]$} \\
\hline & Tissues and cells & Circ_001569 & $\operatorname{miR}-145$ & E2F5, BAG4, FMNL2 & {$[32]$} \\
\hline Cholangiocarcinoma & Tissues & CDR1as & - & - & {$[26]$} \\
\hline \multirow{3}{*}{ Esophageal squamous cell carcinoma } & Tissues & CDR1as & miR-876-5p & MAGE-A family & {$[23]$} \\
\hline & Tissues & circ-ITCH & miR-7 & Wnt/ $\beta$-catenin & {$[24]$} \\
\hline & Tissues and cells & CDR1as & miR-135a & $\mathrm{P} 21$ & {$[25]$} \\
\hline Bladder cancer & Tissues and cells & circTCF25 & $\begin{array}{l}\text { miR-103a-3p, } \\
\text { miR-107 }\end{array}$ & CDK6 & {$[28]$} \\
\hline Osteosarcoma & cell lines, tissues and plasmas & circ-HIPK3 & - & - & [27] \\
\hline \multirow{2}{*}{ Gastric cancer } & Tissues and plasma & hsa_circ_002059 & - & - & {$[29]$} \\
\hline & Tissues and cell lines & CircRNA_100269 & miR-630 & - & {$[30]$} \\
\hline Oral squamous cell carcinomas & Tissues & CircRNA_100290 & miR-29b family & CDK6 & {$[31]$} \\
\hline
\end{tabular}

SRY, sex-determining region Y; RhoC, Ras homolog gene family member C; CCNE1, Cyclin E1; PIK3CD, phosphoinositide 3-kinase catalytic subunit delta; p70S6K, p70S6 kinase; EGFR, Epidermal growth factor receptor; IGF-1R, Insulin-like growth factor 1 receptor; E2F5, E2F Transcription Factor 5 Protein; BAG-4, BCL2-associated athanogene 4; FMNL2, Formin-like 2; MAGE-A family, melanoma-antigen family A; CDK6, cyclin-dependent kinase 6.

myocardial infarction ${ }^{[39]}$.

A circRNA termed "heart-related circRNA" (HRCR) has been proven to function as an endogenous miR-223 sponge to inhibit cardiac hypertrophy and heart failure, providing an attractive target for the diagnosis and treatment of these disorders ${ }^{[40]}$. The circular antisense non-coding RNA in the INK4 locus (circANRIL) is located at a position of atherosclerotic cardiovascular disease on chromosome 9p21, conferring atheroprotection by binding to pescadillo homolog 1 (PES1), an essential 60S-preribosomal assembly factor, thereby impairing exonuclease-mediated pre-rRNA processing and ribosome biogenesis in vascular smooth muscle cells and macrophages [41]. CircRNAs related to cardiovascular diseases and their potential targets are displayed in Table 3.

\section{CIRCRNAS AND OTHER COMMON DIS- ORDERS}

Apart from these cancers and disorders of the nervous and cardiovascular systems, many other common diseases have been shown to be associated with circRNAs (Table 4). A strong interaction pair, CDR1as/miR-7, was identified in islet cells, and the overexpression of CDR1as was shown to enhance insulin content and secretion, contributing to the improvement of $\beta$ cell function in diabetes ${ }^{[42]}$. In the peripheral blood of type 2 diabetes mellitus patients, 489 circRNAs were discovered to be differentially expressed, including 78 upregulated and 411 downregulated. Among them, hsa_circ_0054633 showed the highest value as a therapeutic target in prediabetes and type 2 diabetes mellitus ${ }^{[43]}$. Through the study of sera from diabetic retinopathy patients via circular microarray, 30 circRNAs were observed to be markedly upregulated ${ }^{[44]}$. Subsequently, circ_0005015 was verified as being upregulated in the plasma, vitreous samples, and fibrovascular membranes of diabetic retinopathy patients and to facilitate retinal endothelial angiogenic function by targeting miR-519d$3 p{ }^{[45]}$. These findings suggest the potential function of circRNAs in the pathogenesis of diabetic retinopathy through interaction with miRNAs.

The function of the chondrocyte extracellular matrix (ECM)-related circRNA (circRNA-CER) in osteoarthritis has also been unraveled. It competes for miR-136 with MMP13 to participate in the process of chondrocyte ECM degradation ${ }^{[46]}$. Hsa_circ_0005105 also promotes chondrocyte ECM degradation by sponging miR-26a and regulating nicotinamide phosphoribosyltransferase (NAMPT) ${ }^{[47]}$. Their involvement in pathogenesis earns these two circRNAs the biological marker property in osteoarthritis and other orthopedic diseases.

In the livers of nonalcoholic steatohepatitis model mice, 69 upregulated and 63 downregulated circRNAs have been authenticated, and four circRNA-miRNA-mRNA pathways have been constructed (Table 4), providing candidate targets for nonalcoholic steatohepatitis treatment ${ }^{[48]}$. Finally, given the roles of circRNAs in sponging miRNAs and the implication of miRNAs 
Table 3: CircRNAs involved in cardiovascular diseases.

\begin{tabular}{|c|c|c|c|c|c|}
\hline & Sites or samples & CircRNAs & MiRNAs & Potential targets & References \\
\hline \multirow{2}{*}{ Myocardial fibrosis } & Myocardium & circRNA_000203 & miR-26b-5p & Col1a2 and CTGF & {$[34]$} \\
\hline & Myocardium & circRNA_010567 & miR-141 & TGF- $\beta 1$ & {$[35]$} \\
\hline \multirow{2}{*}{ Myocardial infarction } & Peripheral blood & MICRA & - & - & {$[37,38]$} \\
\hline & Mouse model, cardiomyocytes & CDR1as & $\operatorname{miR}-7$ & PARP and SP1 & [39] \\
\hline Cardiac hypertrophy & Mouse heart & HRCR & $\operatorname{miR}-223$ & ARC & {$[40]$} \\
\hline Atherosclerosis & Tissues & circANRIL & - & PES1 & {$[41]$} \\
\hline
\end{tabular}

MICRA, myocardial infarction-associated circRNA; HRCR, heart-related circRNA; Col1a2, Collagen, type I, alpha 2; CTGF, Connective tissue growth factor; PARP, Poly(ADP-Ribose) polymerases; SP1, specificity protein 1; ARC, Apoptosis repressor with caspase recruitment domain; PES1, pescadillo homologue 1.

in the initiation and progression of systemic lupus erythematosus (SLE), circRNAs have been conjectured to play a role in SLE ${ }^{[49]}$, and this must be examined closely in future studies.

\section{BIOMARKER ROLES OF CIRCRNAS IN EARLY DIAGNOSIS, TARGETED THER- APY, AND PROGNOSIS}

Biomarkers are officially defined as characteristics that are objectively measured and evaluated as indicators of normal biological processes, pathogenic processes, or pharmacologic responses to a therapeutic intervention [50]. Due to their indicative role in various biological and pathogenic processes, biomarkers are widely identified in many human diseases for their value in early diagnosis, personalized treatment, pharmaceutical research, and prognosis [51-54] The ncRNAs, such as the common lncRNAs and miRNAs, are a large class of macromolecular biomarkers that have attracted much attention. Recently, circRNAs have become potential new biomarkers for the processes of various disorders because of their abundance, conservation, and stagespecific and tissue-specific expression ${ }^{[6]}$. CircRNAs are usually observed to be up- or downregulated in various specimens from diseased patients, such as tissue, plasma, peripheral blood, and saliva, which are easy and suitable to obtain for quantification via painless and minimally invasive methods ${ }^{[55]}$.

The clearest case is the circRNA CDR1as, which can become a biomarker for neurodegenerative disorders [11], hepatocellular carcinoma [19], and myocardial infarction ${ }^{[39]}$. In addition, a prognostic value analysis and a correlation analysis between CDR1as level and overall patient survival demonstrated that CDR1as expression could be considered an independent prognostic biomarker for cholangiocarcinoma [26]. The circRNA MICRA improved risk classification after myocardial infarction, supporting its value as a novel biomarker in future prognostication strategies ${ }^{[38]}$. The inhibitory effect of circ-HIPK3 overexpression on the proliferation, invasion, and migration of osteosarcoma cells makes it a promising diagnostic biomarker and treatment target in osteosarcoma ${ }^{[27]}$. In gastric cancer, a low expression level of hsa_circ_002059 was noted and significantly associated with advanced TNM stage, distal metastasis, male, and age, suggesting that this circRNA offers great potential as a stable biomarker for the diagnosis of gastric carcinoma ${ }^{[29]}$. Finally, circRNAs are enriched and stable in exosomes, thus presenting a promising biomarker for cancer diagnosis ${ }^{[56]}$.

We have demonstrated that circRNAs play critical roles in human disease pathogenesis by interacting with miRNAs. We have also shown that the abnormal expression of circRNAs in pathological conditions

Table 4: CircRNAs and other common diseases.

\begin{tabular}{|c|c|c|c|c|c|}
\hline & Sites or samples & CircRnAs & MiRNAs & Potential targets & References \\
\hline \multirow{2}{*}{ Diabetes } & Islet cells & CDR1as & miR-7 & Myrip, Pax6 & {$[42]$} \\
\hline & peripheral blood & hsa_circ_0054633 & - & - & {$[43]$} \\
\hline Diabetic retinopathy & Plasma and tissues & circ_0005015 & miR-519d-3p & MMP-2, XIAP, and STAT3 & {$[45]$} \\
\hline \multirow{2}{*}{ Osteoarthritis } & Cartilage & circRNA-CER & miR-136 & MMP13 & {$[46]$} \\
\hline & Chondrocytes & hsa_circ_0005105 & miR-26a & NAMPT & {$[47]$} \\
\hline \multirow{2}{*}{ Nonalcoholic steatohepatitis } & \multirow{2}{*}{ Liver tissues } & circRNA_002581 & miR-122 & Slc1a5, Plp2, and Cpeb1 & {$[48]$} \\
\hline & & circRNA_007585 & miR-326 & UCP2 & {$[48]$} \\
\hline
\end{tabular}

Pax6, Paired box 6; MMP-2, Matrix metalloproteinase-2; XIAP, X-linked inhibitor of apoptosis; STAT3, signal transducers and activator of transcription 3; MMP13, Matrix metalloproteinase 13; NAMPT, nicotinamide phosphoribosyltransferase; Slc1a5, solute carrier family A1 member 5; Plp2, proteolipid protein 2; Cpeb1, cytoplasmic polyadenylation element binding protein 1; UCP2, uncoupling protein 2. 
makes them optimal clinical therapeutic targets and biomarkers for disease diagnosis and therapeutic and prognostic evaluation. As future sequencing techniques and data analysis methods evolve, we can expect more circRNAs to be exploited in various tissues and organs. Although the sponge activities and biomarker prospects of circRNAs have been fully discussed, some issues must urgently be resolved before their formal utility. Firstly, the nomenclature for circRNAs has not been standardized, and the current databases are awaiting integration. Secondly, additional bioinformatics methods must be developed for data processing. Finally, the biogenesis of circRNAs and their pathological pathways have not yet been thoroughly elucidated. With the prompt resolution of the above problems, we may anticipate the wide application of circRNAs as therapeutic targets and diagnostic biomarkers, which may lead to breakthroughs from evidence-based to precision medicine.

\section{REFERENCES}

1. Bolha, L., Ravnik-Glavac, M., and Glavac, D. (2017) Circular RNAs: Biogenesis, Function, and a Role as Possible Cancer Biomarkers. Int J Genomics 2017, 6218353

2. Chen, L. L., and Yang, L. (2015) Regulation of circRNA biogenesis. RNA Biol 12, 381-388

3. Qu, S., Yang, X., Li, X., Wang, J., Gao, Y., Shang, R., Sun, W., Dou, K., and Li, H. (2015) Circular RNA: A new star of noncoding RNAs. Cancer Lett 365, 141-148

4. Hansen, T. B., Kjems, J., and Damgaard, C. K. (2013) Circular RNA and miR-7 in cancer. Cancer Res 73, 5609-5612

5. 5.Dey, B. K., Mueller, A. C., and Dutta, A. (2014) Long non-coding RNAs as emerging regulators of differentiation, development, and disease. Transcription 5, e944014

6. Kulcheski, F. R., Christoff, A. P., and Margis, R. (2016) Circular RNAs are miRNA sponges and can be used as a new class of biomarker. J Biotechnol 238, 42-51

7. van Rossum, D., Verheijen, B. M., and Pasterkamp, R. J. (2016) Circular RNAs: Novel Regulators of Neuronal Development. Front Mol Neurosci 9, 74

8. Rybak-Wolf, A., Stottmeister, C., Glazar, P., Jens, M., Pino, N., Giusti, S., Hanan, M., Behm, M., Bartok, O., Ashwal-Fluss, R., Herzog, M., Schreyer, L., Papavasileiou, P., Ivanov, A., Ohman, M., Refojo, D., Kadener, S., and Rajewsky, N. (2015) Circular RNAs in the Mammalian Brain Are Highly Abundant, Conserved, and Dynamically Expressed. Mol Cell 58, 870-885

9. Guo, J. U., Agarwal, V., Guo, H., and Bartel, D. P. (2014) Expanded identification and characterization of mammalian circular RNAs. Genome Biol 15, 409

10. Barbagallo, D., Condorelli, A., Ragusa, M., Salito, L., Sammito, M., Banelli, B., Caltabiano, R., Barbagallo, G., Zappala, A., Battaglia, R., Cirnigliaro, M., Lanzafame, S.,
Vasquez, E., Parenti, R., Cicirata, F., Di Pietro, C., Romani, M., and Purrello, M. (2016) Dysregulated miR-671-5p / CDR1-AS / CDR1 / VSNL1 axis is involved in glioblastoma multiforme. Oncotarget 7, 4746-4759

11. Lukiw, W. J. (2013) Circular RNA (circRNA) in Alzheimer's disease (AD). Front Genet 4, 307

12. 12.Ghosal, S., Das, S., Sen, R., Basak, P., and Chakrabarti, J. (2013) Circ2Traits: a comprehensive database for circular RNA potentially associated with disease and traits. Front Genet 4, 283

13. Piwecka, M., Glazar, P., Hernandez-Miranda, L. R., Memczak, S., Wolf, S. A., Rybak-Wolf, A., Filipchyk, A., Klironomos, F., Cerda Jara, C. A., Fenske, P., Trimbuch, T., Zywitza, V., Plass, M., Schreyer, L., Ayoub, S., Kocks, C., Kuhn, R., Rosenmund, C., Birchmeier, C., and Rajewsky, N. (2017) Loss of a mammalian circular RNA locus causes miRNA deregulation and affects brain function. Science 357

14. Zhou, J., Xiong, Q., Chen, H., Yang, C., and Fan, Y. (2017) Identification of the Spinal Expression Profile of Non-coding RNAs Involved in Neuropathic Pain Following Spared Nerve Injury by Sequence Analysis. Front Mol Neurosci 10,91

15. Lin, S. P., Ye, S., Long, Y., Fan, Y., Mao, H. F., Chen, M. T., and Ma, Q. J. (2016) Circular RNA expression alterations are involved in OGD/R-induced neuron injury. Biochem Biophys Res Commun 471, 52-56

16. Teng, L., Chen, Y., Chen, H., He, X., Wang, J., Peng, Y., Duan, H., Li, H., Lin, D., and Shao, B. (2017) Circular RNA hsa_ circ_0021001 in peripheral blood: a potential novel biomarker in the screening of intracranial aneurysm. Oncotarget 8, 107125-107133

17. Hansen, T. B., Jensen, T. I., Clausen, B. H., Bramsen, J. B., Finsen, B., Damgaard, C. K., and Kjems, J. (2013) Natural RNA circles function as efficient microRNA sponges. Nature 495, 384-388

18. Zhao, Z. J., and Shen, J. (2017) Circular RNA participates in the carcinogenesis and the malignant behavior of cancer. RNA Biol 14, 514-521

19. Yu, L., Gong, X., Sun, L., Zhou, Q., Lu, B., and Zhu, L. (2016) The Circular RNA Cdr1as Act as an Oncogene in Hepatocellular Carcinoma through Targeting miR-7 Expression. PLoS One 11, e0158347

20. Xu, L., Zhang, M., Zheng, X., Yi, P., Lan, C., and Xu, M. (2017) The circular RNA ciRS-7 (Cdr1as) acts as a risk factor of hepatic microvascular invasion in hepatocellular carcinoma. J Cancer Res Clin Oncol 143, 17-27

21. Yang, X., Xiong, Q., Wu, Y., Li, S., and Ge, F. (2017) Quantitative Proteomics Reveals the Regulatory Networks of Circular RNA CDR1as in Hepatocellular Carcinoma Cells. J Proteome Res 16, 3891-3902

22. Tang, W., Ji, M., He, G., Yang, L., Niu, Z., Jian, M., Wei, Y., Ren, L., and Xu, J. (2017) Silencing CDR1as inhibits colorectal cancer progression through regulating microRNA-7. Onco Targets Ther 10, 2045-2056 
23. Sang, M., Meng, L., Sang, Y., Liu, S., Ding, P., Ju, Y., Liu, F., Gu, L., Lian, Y., Li, J., Wu, Y., Zhang, X., and Shan, B. (2018) Circular RNA ciRS-7 accelerates ESCC progression through acting as a miR-876-5p sponge to enhance MAGE-A family expression. Cancer Lett 426, 37-46

24. Li, F., Zhang, L., Li, W., Deng, J., Zheng, J., An, M., Lu, J., and Zhou, Y. (2015) Circular RNA ITCH has inhibitory effect on ESCC by suppressing the Wnt/beta-catenin pathway. Oncotarget 6, 6001-6013

25. Li, P., Yang, X., Yuan, W., Yang, C., Zhang, X., Han, J., Wang, J., Deng, X., Yang, H., Li, P., Tao, J., Lu, Q., and Gu, M. (2018) CircRNA-Cdr1as Exerts Anti-Oncogenic Functions in Bladder Cancer by Sponging MicroRNA-135a. Cell Physiol Biochem 46, 1606-1616

26. Jiang, X. M., Li, Z. L., Li, J. L., Xu, Y., Leng, K. M., Cui, Y. F., and Sun, D. J. (2018) A novel prognostic biomarker for cholangiocarcinoma: circRNA Cdr1as. Eur Rev Med Pharmacol Sci 22, 365-371

27. Xiao-Long, M., Kun-Peng, Z., and Chun-Lin, Z. (2018) Circular RNA circ_HIPK3 is down-regulated and suppresses cell proliferation, migration and invasion in osteosarcoma. J Cancer 9, 1856-1862

28. Zhong, Z., Lv, M., and Chen, J. (2016) Screening differential circular RNA expression profiles reveals the regulatory role of circTCF25-miR-103a-3p/miR-107-CDK6 pathway in bladder carcinoma. Sci Rep 6, 30919

29. Li, P., Chen, S., Chen, H., Mo, X., Li, T., Shao, Y., Xiao, B., and Guo, J. (2015) Using circular RNA as a novel type of biomarker in the screening of gastric cancer. Clin Chim Acta 444, 132-136

30. Zhang, Y., Liu, H., Li, W., Yu, J., Li, J., Shen, Z., Ye, G., Qi, X., and Li, G. (2017) CircRNA_100269 is downregulated in gastric cancer and suppresses tumor cell growth by targeting miR-630. Aging (Albany NY) 9, 1585-1594

31. Chen, L., Zhang, S., Wu, J., Cui, J., Zhong, L., Zeng, L., and Ge, S. (2017) circRNA_100290 plays a role in oral cancer by functioning as a sponge of the miR-29 family. Oncogene 36, 4551-4561

32. Xie, H., Ren, X., Xin, S., Lan, X., Lu, G., Lin, Y., Yang, S., Zeng, Z., Liao, W., Ding, Y. Q., and Liang, L. (2016) Emerging roles of circRNA_001569 targeting miR-145 in the proliferation and invasion of colorectal cancer. Oncotarget 7, 26680-26691

33. Ahearn, J., Shields, K. J., Liu, C. C., and Manzi, S. (2015) Cardiovascular disease biomarkers across autoimmune diseases. Clin Immunol 161, 59-63

34. Tang, C. M., Zhang, M., Huang, L., Hu, Z. Q., Zhu, J. N., Xiao, Z., Zhang, Z., Lin, Q. X., Zheng, X. L., Yang, M., Wu, S. L., Cheng, J. D., and Shan, Z. X. (2017) CircRNA_000203 enhances the expression of fibrosis-associated genes by derepressing targets of miR-26b-5p, Col1a2 and CTGF, in cardiac fibroblasts. Sci Rep 7, 40342

35. Zhou, B., and Yu, J. W. (2017) A novel identified circular RNA, circRNA_010567, promotes myocardial fibrosis via suppressing miR-141 by targeting TGF-beta1. Biochem Biophys Res Commun 487, 769-775

36. Lin, F., Zhao, G. A., Chen, Z. G., Wang, X. H., Lu, F. H., Zhang, Y. C., Cai, R. Y., Liang, W. Q., Li, J. H., Li, M., Zhang, G. H., and Yang, Y. M. (2018) [Network correlation of circRNA-miRNA and the possible regulatory mechanism in acute myocardial infarction]. Zhonghua Yi Xue Za Zhi 98, 851-854

37. Vausort, M., Salgado-Somoza, A., Zhang, L., Leszek, P., Scholz, M., Teren, A., Burkhardt, R., Thiery, J., Wagner, D. R., and Devaux, Y. (2016) Myocardial Infarction-Associated Circular RNA Predicting Left Ventricular Dysfunction. J Am Coll Cardiol 68, 1247-1248

38. Salgado-Somoza, A., Zhang, L., Vausort, M., and Devaux, Y. (2017) The circular RNA MICRA for risk stratification after myocardial infarction. Int J Cardiol Heart Vasc 17, 33-36

39. Geng, H. H., Li, R., Su, Y. M., Xiao, J., Pan, M., Cai, X. X., and Ji, X. P. (2016) The Circular RNA Cdr1as Promotes Myocardial Infarction by Mediating the Regulation of miR-7a on Its Target Genes Expression. PLoS One 11, e0151753

40. Wang, K., Long, B., Liu, F., Wang, J. X., Liu, C. Y., Zhao, B., Zhou, L. Y., Sun, T., Wang, M., Yu, T., Gong, Y., Liu, J., Dong, Y. H., Li, N., and Li, P. F. (2016) A circular RNA protects the heart from pathological hypertrophy and heart failure by targeting miR-223. Eur Heart J 37, 2602-2611

41. Holdt, L. M., Stahringer, A., Sass, K., Pichler, G., Kulak, N. A., Wilfert, W., Kohlmaier, A., Herbst, A., Northoff, B. H., Nicolaou, A., Gabel, G., Beutner, F., Scholz, M., Thiery, J., Musunuru, K., Krohn, K., Mann, M., and Teupser, D. (2016) Circular non-coding RNA ANRIL modulates ribosomal RNA maturation and atherosclerosis in humans. Nat Commun 7, 12429

42. Xu, H., Guo, S., Li, W., and Yu, P. (2015) The circular RNA Cdr1as, via miR-7 and its targets, regulates insulin transcription and secretion in islet cells. Sci Rep 5, 12453

43. Zhao, Z., Li, X., Jian, D., Hao, P., Rao, L., and Li, M. (2017) Hsa_circ_0054633 in peripheral blood can be used as a diagnostic biomarker of pre-diabetes and type 2 diabetes mellitus. Acta Diabetol 54, 237-245

44. Gu, Y., Ke, G., Wang, L., Zhou, E., Zhu, K., and Wei, Y. (2017) Altered Expression Profile of Circular RNAs in the Serum of Patients with Diabetic Retinopathy Revealed by Microarray. Ophthalmic Res 58, 176-184

45. Zhang, S. J., Chen, X., Li, C. P., Li, X. M., Liu, C., Liu, B. H., Shan, K., Jiang, Q., Zhao, C., and Yan, B. (2017) Identification and Characterization of Circular RNAs as a New Class of Putative Biomarkers in Diabetes Retinopathy. Invest Ophthalmol Vis Sci 58, 6500-6509

46. Liu, Q., Zhang, X., Hu, X., Dai, L., Fu, X., Zhang, J., and Ao, Y. (2016) Circular RNA Related to the Chondrocyte ECM Regulates MMP13 Expression by Functioning as a MiR136 'Sponge' in Human Cartilage Degradation. Sci Rep 6, 22572

47. Wu, Y., Zhang, Y., Zhang, Y., and Wang, J. J. (2017) Cir- 
cRNA hsa_circ_0005105 upregulates NAMPT expression and promotes chondrocyte extracellular matrix degradation by sponging miR-26a. Cell Biol Int 41, 1283-1289

48. Jin, X., Feng, C. Y., Xiang, Z., Chen, Y. P., and Li, Y. M. (2016) CircRNA expression pattern and circRNA-miRNA-mRNA network in the pathogenesis of nonalcoholic steatohepatitis. Oncotarget 7, 66455-66467

49. Li, L. J., Huang, Q., Pan, H. F., and Ye, D. Q. (2016) Circular RNAs and systemic lupus erythematosus. Exp Cell Res 346, 248-254

50. Zhao, X., Modur, V., Carayannopoulos, L. N., and Laterza, O. F. (2015) Biomarkers in Pharmaceutical Research. Clin Chem 61, 1343-1353

51. Tsuchiya, N., Sawada, Y., Endo, I., Saito, K., Uemura, Y., and Nakatsura, T. (2015) Biomarkers for the early diagnosis of hepatocellular carcinoma. World J Gastroenterol 21, 10573-10583

52. Kalia, M. (2015) Biomarkers for personalized oncology: recent advances and future challenges. Metabolism 64, S16-21

53. Hata, S. (2015) [Molecular Pathogenesis of Sporadic Alzheimer's Disease (AD) and Pharmaceutical Research to Develop a Biomarker for AD Diagnosis]. Yakugaku Zasshi 135, 1023-1027

54. Esfahani, M., Ataei, N., and Panjehpour, M. (2015) Biomarkers for evaluation of prostate cancer prognosis. Asian Pac J Cancer Prev 16, 2601-2611

55. Memczak, S., Papavasileiou, P., Peters, O., and Rajewsky, N. (2015) Identification and Characterization of Circular RNAs As a New Class of Putative Biomarkers in Human Blood. PLoS One 10, e0141214

56. Li, Y., Zheng, Q., Bao, C., Li, S., Guo, W., Zhao, J., Chen, D., Gu, J., He, X., and Huang, S. (2015) Circular RNA is enriched and stable in exosomes: a promising biomarker for cancer diagnosis. Cell Res 25, 981-984 